\title{
Chipless RFID Reading Method Insensitive to Tag Orientation
}

\author{
Nicolas Barbot, Member, IEEE, Olivier Rance, Member, IEEE, and Etienne Perret, Senior Member, IEEE
}

\begin{abstract}
This paper presents a new reading method which is able to decode the backscattered signal of a chipless tag without any assumption on its orientation. The method is analytically justified by a model and can be applied to any known scatterers or chipless tags composed of multiple resonators. The presented approach is based on two orthogonal measurements in copolarization on the reader part and can be optionally combined with time gating. Finally, read range is not reduced with this method since the entire energy of the chipless tag's antenna mode is exploited by the reader.
\end{abstract}

Index Terms-Chipless RFID, orientation sensor, RCS, scatterer, UWB.

\section{INTRODUCTION}

Chipless RFID is an emergent perspective able to offer new possibilities compared to traditional barcodes or classical chipped RFID. From the RFID point of view, chipless RFID allows reducing the cost of a tag since it does not use any silicon chip. From the barcode point of view, chipless RFID introduces interesting advances such as non-line of sight reading or sensing due to the properties of RF signals.

Since the introduction of this technology, a lot of work has been done, both at tag and reader level, to increase the coding capacity [1], to improve the robustness of the reading [2], or to comply with regulation standards [3]. However, the chipless RFID technology is still limited by technical issues such as reading distance and coding capacity. Orientation is also a key challenge since for most of RFID chipless systems, tag's orientation has to be known by the reader before the interrogation. Considerable effort has been put into the tag design to overcome this limitation both in co-polarization [4][8] and cross-polarization [9], [10], but few papers have addressed this problem from the reader side [11], [12].

In this paper, a new reading method has been introduced to read any chipless tag whatever its orientation. This method alleviates the constraints on the tag design while achieving the orientation independence. Also, performance of the reading method can be improved compared to classical methods since the total energy of the chipless tag's antenna mode is exploited here.

The paper is organized as follows, in Section II, the analytical model is first introduced, then the reading method insensitive to the tag orientation is derived in Section III. Simulations and measurements are respectively presented in Section IV and V. Finally, Section VI concludes the paper.

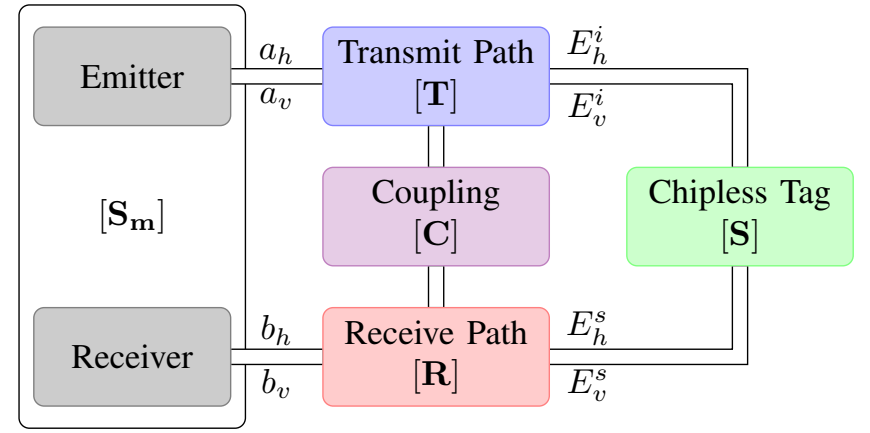

Fig. 1. Channel model of the chipless RFID system for the orientation independent reading method.

Antenna

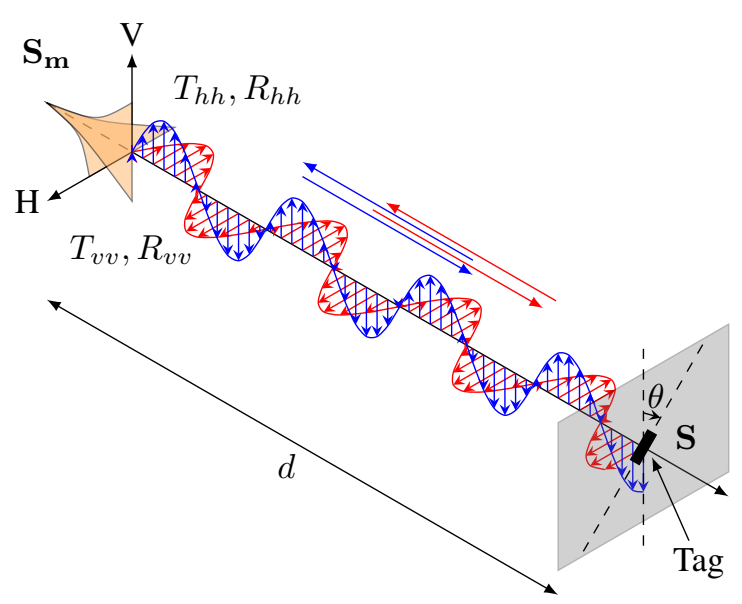

Fig. 2. Principle of the measurement of the proposed method using a dualaccess, dual-polarization antenna.

\section{Analytical Model}

Chipless tag reading can be described using the model introduced in [13] and represented in Fig. 1 for clarity. The S-parameters $\mathbf{S}_{\mathbf{m}}$ measured by the VNA can be expressed as:

$$
\mathbf{S}_{\mathbf{m}}=\mathbf{T} \cdot \mathbf{C} \cdot \mathbf{R}+\mathbf{T} \cdot \mathbf{S} \cdot \mathbf{R}
$$

where $\mathbf{T}$ and $\mathbf{R}$ represent the transmit path and received path, and $\mathbf{C}$ the coupling. $\mathbf{S}$ is the polarization scattering matrix of the tag defined by:

$$
\mathbf{S}=\left[\begin{array}{ll}
S_{v v} & S_{v h} \\
S_{h v} & S_{h h}
\end{array}\right]
$$

The authors are with Univ. Grenoble Alpes, Grenoble INP, LCIS, F-26000 Valence, France. 
and depends on its orientation. Note that each term in (1) is a $2 \times 2$ matrix of complex and frequency dependent parameters.

If we now consider a rotation of the tag by an unknown angle $\theta$ under normal incidence (see Fig. 2), the new parameters of the $\mathbf{S}(\theta)$ matrix are linked to the initial ones by the following expression [14]:

$$
\mathbf{S}(\theta)=\mathbf{\Omega}^{T} \cdot \mathbf{S} \cdot \boldsymbol{\Omega}
$$

where ${ }^{T}$ is the transpose operator and $\Omega$ is a rotation matrix of angle $\theta$ defined by:

$$
\boldsymbol{\Omega}=\left[\begin{array}{cc}
\cos \theta & -\sin \theta \\
\sin \theta & \cos \theta
\end{array}\right]
$$

Note that (3) is general and still valid even if the tag is initally not aligned with the propagation direction.

Classical chipless tags are read in vertical (or horizontal) copolarization. In the following, we suppose that the information is stored in the $S_{v v}$ parameter. Form (3), the expression of the vertical co-polarization term $S_{v v}(\theta)$ can be extracted:

$$
S_{v v}(\theta)=S_{v v} \cos ^{2} \theta+S_{v h} \sin 2 \theta+S_{h h} \sin ^{2} \theta
$$

where the admissible values of $S_{v v}(\theta)$ describes an ellipse in the complex plane as a function of $\theta$ (see Fig. 3). As previously said, in order to achieve an orientation insensitive chipless RFID system, authors have usually focused their studies on the tag design. The main idea can be easily explained using (5), where the design of a chipless tag has to satisfy simultaneously the two conditions $S_{v v}=S_{h h}$ and $S_{v h}=0$ so that $S_{v v}(\theta)=S_{v v}$ which is independent of $\theta$. When these conditions are met, $S_{v v}(\theta)$ and $S_{h h}(\theta)$ are identical (in magnitude and phase) and do not depend anymore on the tag orientation. Note that in this specific case, tag polarization scattering matrix of the tag takes the form $\mathbf{S}=S_{v v} \mathbf{I}$. Graphically, this solution transforms the ellipse into a single point. In practice, these conditions can be satisfied for every structure having a rotational symmetry (which include, triangle, square, pentagon, circular patterns...) [15]. This idea has been applied, even if not clearly stated, in [5]-[7] where authors have designed circular chipless tags and in [4], [8], where triangle, hexagonal and trefoil-shaped resonators have been used. All the mentioned tags are read in co-polarization, and do not have any cross-polarization (as predicted by the presented model). However these geometrical constraints on the tag design to overcome the orientation determination can also affect the selectivity, the RCS level and/or the coding density of the proposed resonators. In this paper, we introduce a new reading method independent from the tag orientation and which can be applied to any chipless tag. This method makes it possible to relax the design constraints of the chipless tag at a price of making two measurements by the reader.

\section{Orientation Insensitive ReAding Method}

From (3), scattering parameters in vertical and horizontal co-polarization can respectively be written as:

$$
\left\{\begin{array}{l}
S_{v v}(\theta)=S_{v v} \cos ^{2} \theta+S_{v h} \sin 2 \theta+S_{h h} \sin ^{2} \theta \\
S_{h h}(\theta)=S_{v v} \sin ^{2} \theta-S_{v h} \sin 2 \theta+S_{h h} \cos ^{2} \theta
\end{array}\right.
$$

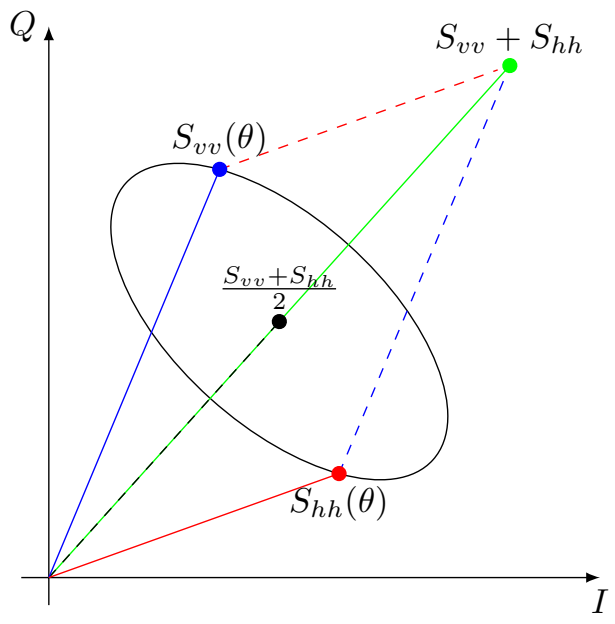

Fig. 3. Admissible values of vertical co-polarization $S_{v v}(\theta)$ and horizontal co-polarization $S_{h h}(\theta)$ and the proposed method (7) in the complex plane for a general scatterer as a function of the orientation $\theta$.

By summing (6a) and (6b), we have:

$$
S_{v v}(\theta)+S_{h h}(\theta)=S_{v v}+S_{h h}
$$

which is independent of $\theta$ [16]. Moreover, (7) holds for any scatterer (i.e., whatever $S_{v v}, S_{h h}$ and $S_{v h}$ values) and applies both on antenna mode and structural mode of the chipless tag. Graphical interpretation can be seen in Fig. 3 where the summation of $S_{v v}(\theta)$ and $S_{h h}(\theta)$ which both lie on an ellipse diameter, corresponds to a point with a magnitude equal to two times the magnitude of the ellipse center. Finally, note that the magnitude of (7) compared to the one obtained with classical reading (i.e., $S_{v v}$ ) can be, superior (when $S_{v v}$ and $S_{h h}$ are added constructively), equal (when $S_{v v} \gg S_{h h}$ ) or inferior (when $S_{v v}$ and $S_{h h}$ have an opposite phase).

From a practical point of view, from (1), assuming a good isolation between horizontal and vertical polarization as in [13], the quantities $S_{v v}(\theta)$ and $S_{h h}(\theta)$ can be linked to the measured S-parameters $S_{11 m}$ and $S_{22 m}$ :

$$
\begin{aligned}
& S_{11 m}=I_{v v}+T_{v v} \cdot R_{v v} \cdot S_{v v}(\theta) \\
& S_{22 m}=I_{h h}+T_{h h} \cdot R_{h h} \cdot S_{h h}(\theta)
\end{aligned}
$$

with $\mathbf{I}=\mathbf{T} \cdot \mathbf{C} \cdot \mathbf{R}$ and where $I_{v v}$ and $I_{h h}$ can be obtained by measuring the empty environment in vertical and horizontal co-polarization respectively. Moreover, if the transmitting/receiving parts are the same in $\mathrm{E}$ and $\mathrm{H}$ planes, i.e., $T_{v v}=T_{h h}$ and $R_{v v}=R_{h h}$, then the quantity:

$$
S_{11 m}-I_{v v}+S_{22 m}-I_{h h}=2 T_{v v} \cdot R_{v v}\left(S_{v v}+S_{h h}\right)
$$

is proportional to (7) and is independent of $\theta$. Note that, to measure vertical and horizontal responses a dual-polarization, dual-access antenna is recommended (see Fig. 2). Also, measurements of $S_{11 m}$ and $S_{22 m}$ have to correspond to the exact same configuration. In practice, these two measurements can also be done sequentially as long as the chipless tag and surrounding environment are not modified during the acquisitions. However the conditions $T_{v v}=T_{h h}$ and $R_{v v}=R_{h h}$ are challenging to obtained on a wide frequency range as 

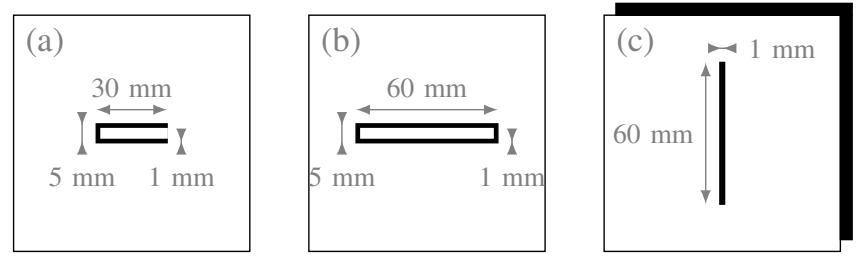

Fig. 4. Resonators used in the study: (a) C-shape resonator, (b) rectangular loop and (c) dipole with ground plane.

needed in chipless RFID. To solve this problem, a calibration measurement is used with a reference target having a rotational symmetry of $90^{\circ}$ (a metallic disk was used in practice). Due to the symmetry, the co-polarization scattering parameters of the disk are characterized by $S_{v v}=S_{h h}=S_{\text {disk }}$. Finally, by noting $S_{X X c}$ the S-parameter measured with the disk, the quantity obtained by:

$$
\frac{S_{11 m}-I_{v v}}{S_{11 c}-I_{v v}}+\frac{S_{22 m}-I_{h h}}{S_{22 c}-I_{h h}}=\frac{S_{v v}+S_{h h}}{S_{\text {disk }}}
$$

is independent of the orientation. Note that this calibration procedure only depends on the antennas used by the reader. Moreover it can be done only once, in an environment that can be different of the one used for tag measurements. Equation (11) has been used in the measurement section.

In order to extract the information of a chipless tag using the presented method, different cases have to be distinguished. For classical tags (like the ones presented in Fig. 4), the information is usually carried by the antenna mode of a single parameter i.e., $S_{v v}$ or $S_{h h}$. That is to say, that all these tags have to be read in a known orientation. Assuming that the information is in the $S_{v v}$ parameter, the proposed method (7) [and (11)] provides a response which contains the summation of $S_{v v}$ (i.e., the antenna mode with a structural mode) and $S_{h h}$ (which contains an additional structural mode without any information). This additional term generally degrades the performance of the reading using (7). However, since duration of the structural mode is usually shorter than the antenna mode, tag ID can be extracted using time gating operation.

For randomly oriented tags, cross-polarization tags [13], [17] or orientation independent tags, the antenna and structural modes are divided on both vertical and horizontal polarization. For these cases, the proposed method (with time gating) enables to recover the total energy of the antenna mode increasing the performance compared to classical readings. Thus the proposed method can be used to decode most of the chipless tags proposed in the literature independently of their orientation. The only notable exception appears for chipless tags which encode information on both polarizations [18], [19]. For these tags, the proposed method breaks the orthogonality of the two polarizations and thus cannot be applied to decode the tag ID.

\section{Simulation}

\section{A. Reading of classical tags with the proposed method}

Scatterers considered in this study are: horizontal C-shape resonator (without ground plane), loop resonator (without ground plane) and vertical dipole resonator with ground plane. Scatterers dimensions are provided in Fig. 4. Simulations have been performed using CST Microwave Studio with time domain solver. All resonators have been designed on a Rogers 4003C $0.8 \mathrm{~mm}$ thickness substrate and are excited with a vertical polarization plane wave. E-field are computed in mono-static vertical and horizontal co-polarization using farfield probes. When time gating has been used, starting time and ending time has been set to $5 \mathrm{~ns}$ and $100 \mathrm{~ns}$ respectively (using a rectangular function). Fig. 5 presents the results obtained by simulation for all the resonators. All the presented resonators present a sharp peak in vertical co-polarization $S_{v v}$ around $2 \mathrm{GHz}$ except the dipole for which we can see a deep due to the complex summation between the resonance and the large structural mode of the ground plane. The position of this peak (or deep for the dipole) along the frequency axis is linked to the geometrical dimensions of the resonator and is classically used to encode the information of a chipless tag. On the other side, $S_{h h}$ does not present any resonance (peak or deep) and depends on the structure of the resonator. For the presented method, we can observe that, for the Cshape resonator, (7) allows to directly extract the peak location without using time gating since at the resonant frequency $S_{v v} \gg S_{h h}$. For the loop resonator and the vertical dipole, $S_{h h}$ can not be neglected in front of $S_{v v}$ at the resonant frequency, thus reading the tag ID using (7) without time gating remains a difficult task. However, by combining (7) and time gating, peaks location can easily be extracted in all cases. Note also that using a classical reading (i.e., using $S_{v v}$ ) requires that the resonator is placed at a correct orientation (if we consider a misorientation of $90^{\circ}$, then $S_{v v}\left(90^{\circ}\right)=S_{h h}\left(0^{\circ}\right)$ and the peak location cannot be estimated.

In the following, a tag composed of eight dipole-like scatterers oriented at $45^{\circ}$ with a single ground plane is considered. This tag has been introduced in [13] for identification purposes and is read classically in cross-polarization. It constitutes an interesting example in this study since it contains a significant structural mode in co-polarization due to its large ground plane. Fig. 6 presents the response of the tag with classical readings, and (7) at $\theta=0^{\circ}$. Note that at this orientation, the dipoles form a $45^{\circ}$ angle to the E-field. We can see that the eight peak locations (i.e., the tag ID) can be correctly estimated directly in cross-polarization and by using time gating in co-polarization and for the proposed method. Moreover, amplitude at the resonant frequencies is $6 \mathrm{~dB}$ higher for (7) compared to the classical methods (in co-polarization or crosspolarization) since all the antenna mode energy is exploited by (7) (and not a single projection as in classical methods). Same results have been also observed for tags based on Cshape and loop resonators. Thus, the proposed method (with time gating) can be used to read most of the chipless tags presented in the literature.

\section{B. Impact of the orientation}

Fig. 7 presents the response of the tag introduced in [13] as a function of its orientation for classical reading in copolarization (dashed line) and by using (7) (plain line). Classical method can read the tag at $0^{\circ}$ and $90^{\circ}$ when the stripes 

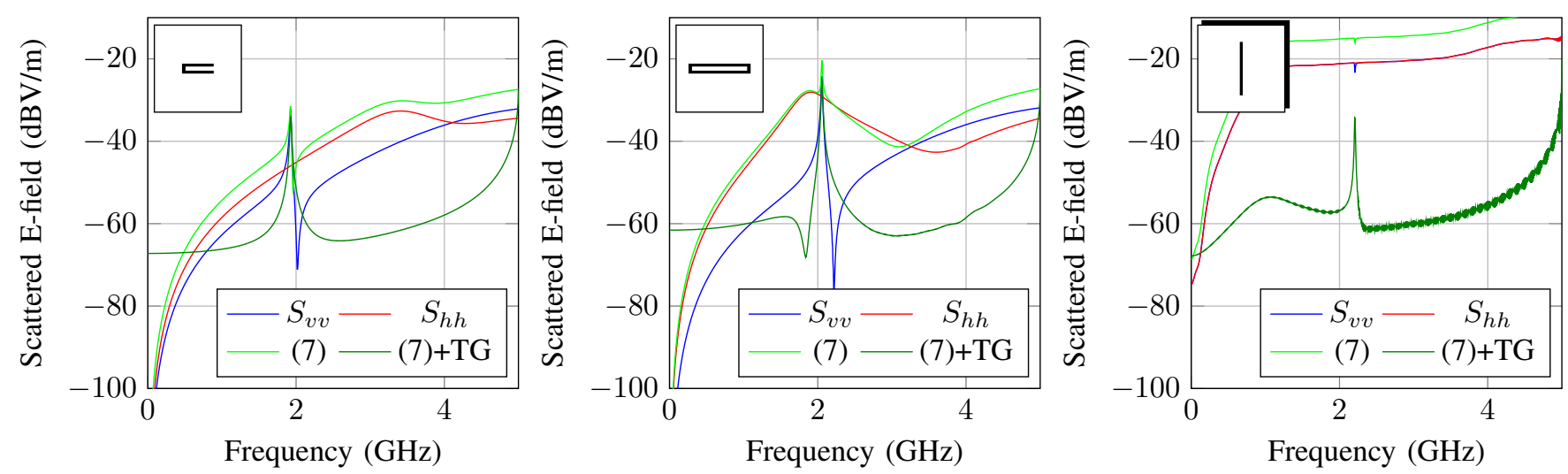

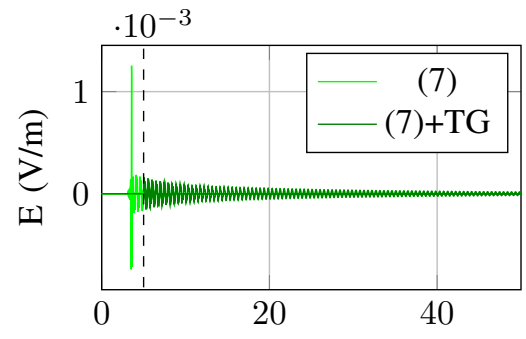

(a) Time (ns)

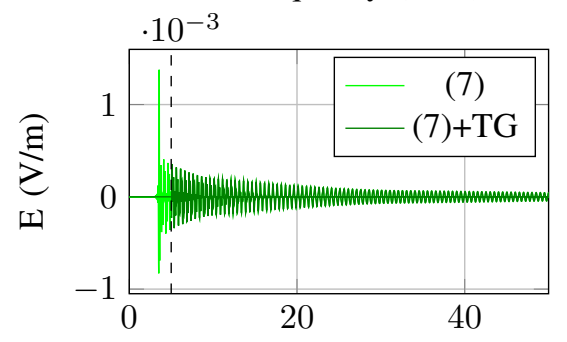

(b) Time (ns)

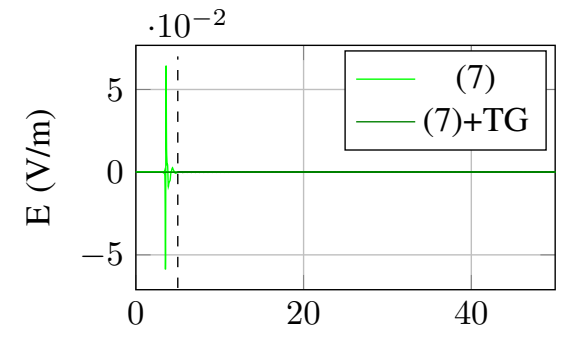

(c) Time (ns)

Fig. 5. Simulated scattered E-field using horizontal and vertical polarizations and (7) (with and without Time Gating) for (a) C-shape resonator, (b) loop resonator and (c) dipole with ground plane.

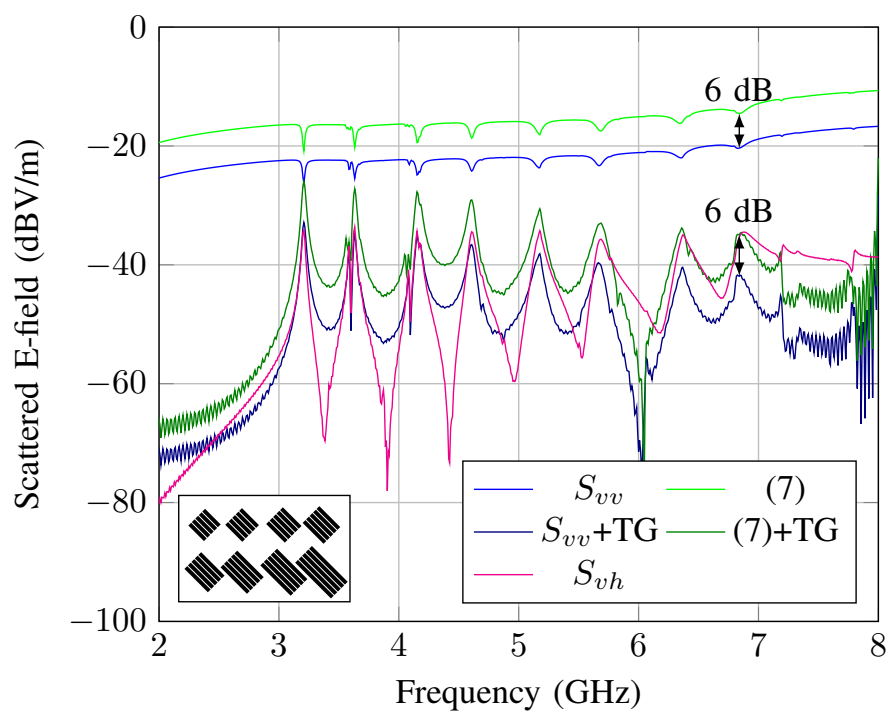

Fig. 6. Simulated scattered E-field for classical readings $\left(S_{v v}\right.$ and $\left.S_{v h}\right)$ and (7) for the tag introduced in [13] at $\theta=0^{\circ}$.

are oriented at $45^{\circ}$ with the E-field (note that at these angles, $S_{h h}(\theta)$ contains half of the antenna mode energy). At $\theta=45^{\circ}$, the amplitude of the peaks tends to 0 since the stripes are perpendicular to the E-field. Note that for this specific orientation, the tag cannot be read anymore by the classical method. Finally for $\theta=-45^{\circ}$ and at the resonant frequencies, (7) and $S_{v v}$ have the same magnitude. On the other side, (7) provides a response which is independent of the tag orientation. It is important to notice that time gating applied on (7) preserves

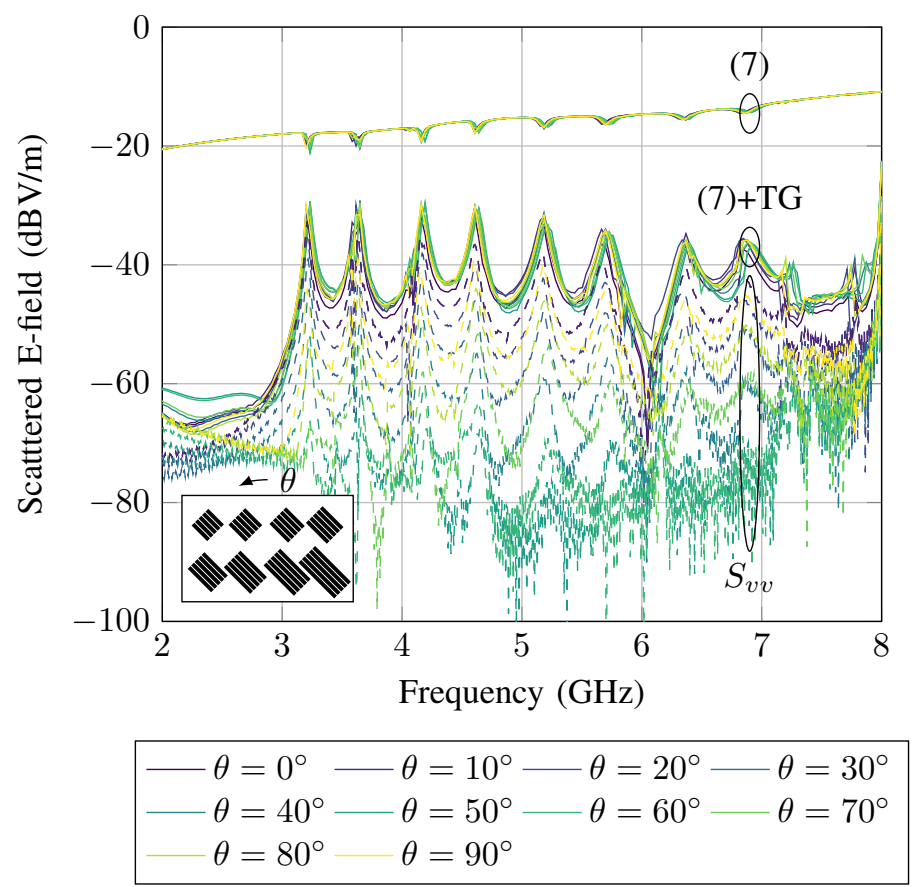

Fig. 7. Simulated scattered E-field obtained using (7) without and with time gating (plain line) and classical reading with time gating (dashed line) for tag introduced in [13] as a function of the orientation.

the orientation independence since it is a linear operation and allows to easily decode the tag whatever its orientation. Maximal variation between backscattered E-field using (7) for all the orientations is respectively $0.37 \mathrm{~dB}$ before time gating and $4.77 \mathrm{~dB}$ after, in the tag operation bandwidth. Note that for 


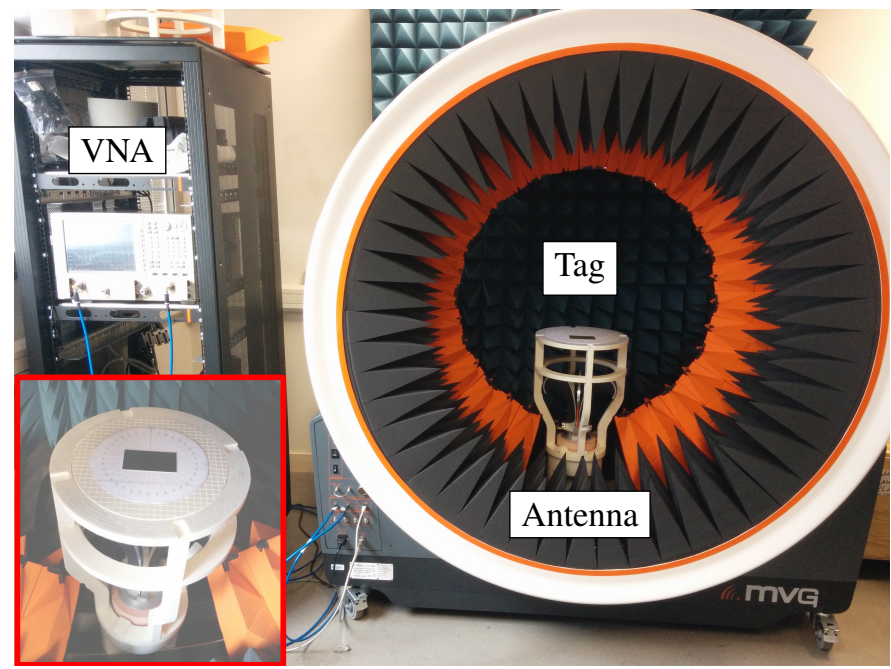

Fig. 8. Measurement bench used for the orientation insensitive reading method.

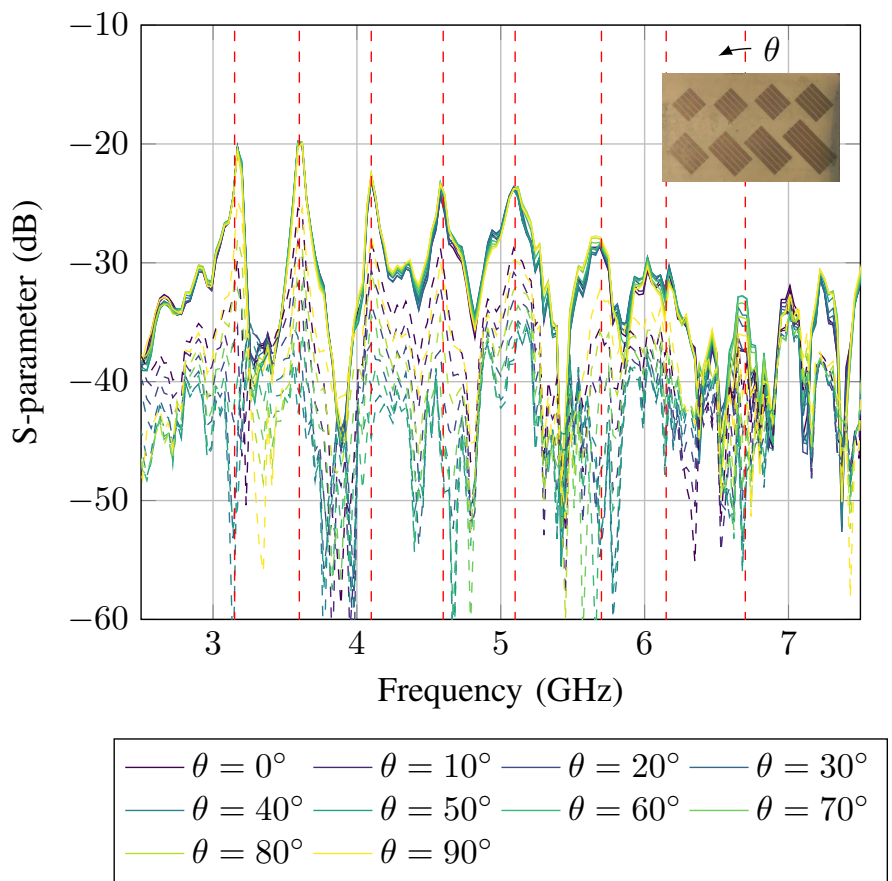

Fig. 9. Measured S-parameters obtained using (11) with time gating (plain line) and classical reading $S_{v v}$ with time gating (dashed line) for tag introduced in [13] at a distance of $d=15 \mathrm{~cm}$ as a function of the orientation.

classical reading, $S_{v v}$ undergoes variation higher than $50 \mathrm{~dB}$ as a function of $\theta$. Finally, for the presented tag at $\theta=0$, the total energy of the antenna mode contained in $S_{v v}$ and $S_{h h}$ is exploited by the proposed method which permits to increase the received energy compared to classical readings.

\section{Measurement}

After introducing the measurement bench, this section presents the performance of the proposed approach compared to the classical ones. Moreover a study on the distance in real environment is provided at the end of the section.

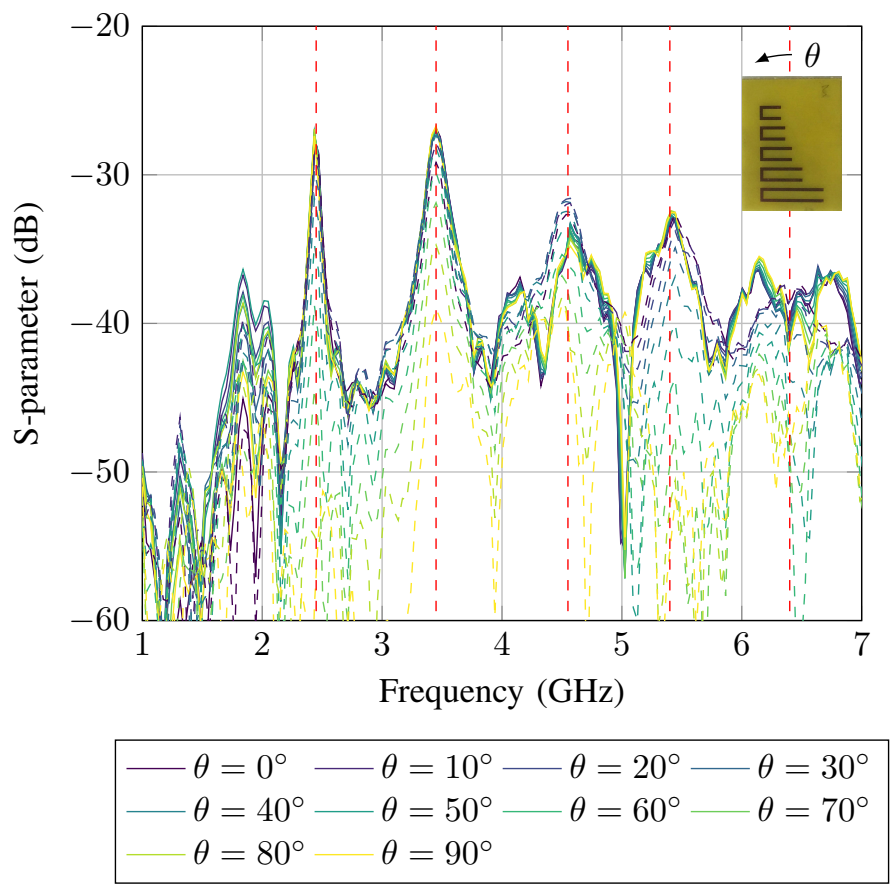

Fig. 10. Measured S-parameters obtained using (11) with time gating (plain line) and classical reading $S_{v v}$ with time gating (dashed line) for tag composed of C-shape resonators [20] at a distance of $d=15 \mathrm{~cm}$ as a function of the orientation.

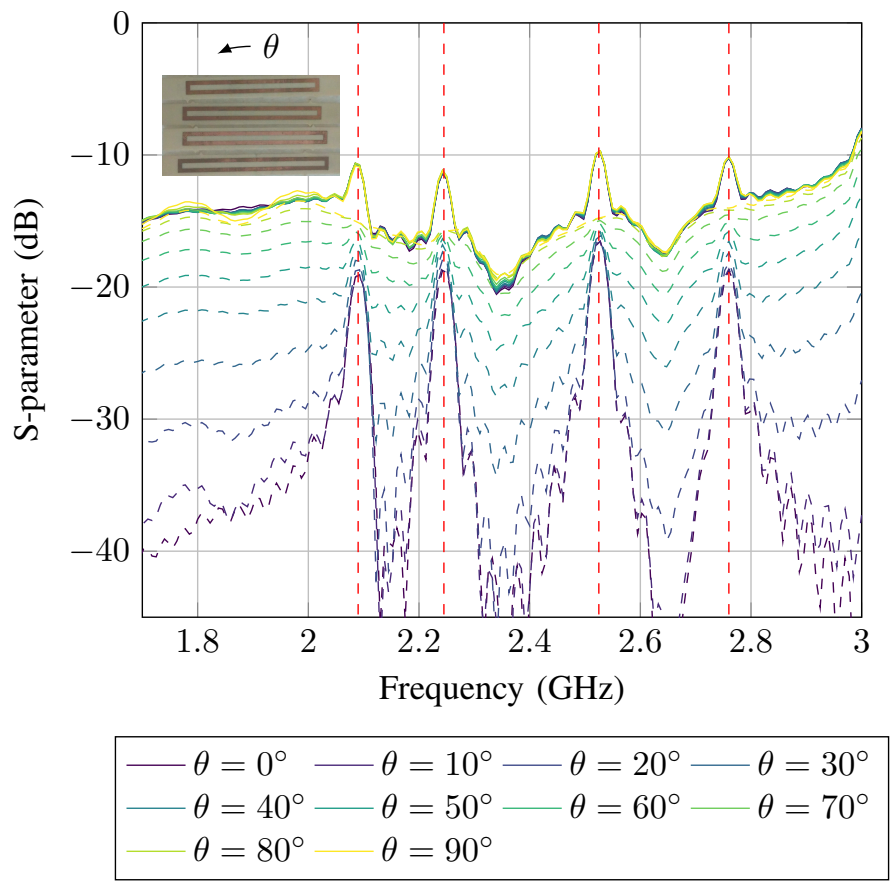

Fig. 11. Measured S-parameters obtained using (11) with time gating (plain line) and classical reading $S_{v v}$ with time gating (dashed line) for tag composed of loop resonators at a distance of $d=15 \mathrm{~cm}$ as a function of the orientation. 


\section{A. Impact of the orientation}

Performance of the proposed approach has been evaluated experimentally by rotating a chipless tag and by using (11). Results have also been compared to the classical reading method which only considers a single polarization. Measurement bench is presented in Fig. 8 and is composed of a VNA (Agilent PNA N5222A) connected to a dual-access dualpolarization antenna (Satimo QH600) to port 1 and 2. This configuration makes it possible to determine $S_{v v}$ and $S_{h h}$ by measuring $S_{11 m}$ and $S_{22 m}$ respectively, with the VNA. Measurements are performed in a semi-anechoic environment (MVG Starlab). The tag is positioned above the antenna at a distance $d=15 \mathrm{~cm}$ using a foam support and is rotated accurately along normal incidence using a stepper motor. VNA settings have been set to a frequency range of $2.5 \mathrm{GHz}-$ $8 \mathrm{GHz}$, a number of points of 1001, and an IF bandwidth of $100 \mathrm{kHz}$. Also, no averaging or calibration has been used. This configuration corresponds to a sweep time of $7.25 \mathrm{~ms}$. Total measurement time $\left(S_{v v}\right.$ and $\left.S_{h h}\right)$ is thus done in $14.5 \mathrm{~ms}$. Note that, during this time, the tag and the surrounding environment have to remain motionless. Fig. 9 presents the results for the tag introduced in [13] for classical reading in co-polarization and by using (11) both with time gating. For comparison purposes, time gating parameters are identical for the classical reading and for the proposed method and has been set to $2.6 \mathrm{~ns}$ and $30 \mathrm{~ns}$ for respectively starting time and ending time. At the correct orientation $\theta=0^{\circ}$ (and $\theta=90^{\circ}$ ) only the six first resonators can be read with the classical method whereas (11) can be used to decode the seven first resonators. However when orientation is changed, the classical method can not be used easily even for low frequency resonators. On the other side, (11) provides a response which is independent of the orientation and $6 \mathrm{~dB}$ higher in magnitude at the resonant frequencies compared to the classical reading at $\theta=0$ since $S_{v v}(0) \approx S_{h h}(0)$. Also maximal variation between measurements using (11) in semi-anechoic environment for all the orientation is equal to $0.24 \mathrm{~dB}$ before time gating and $1.74 \mathrm{~dB}$ after, which shows the robustness of the approach.

The same bench has also been used to read tags without ground plane. Two tags have been considered here, the first one is based on five C-shape resonators (see Fig. 10) and has been introduced in [20], whereas the second one is based on four loop resonators (see Fig. 11). Fig. 10 presents the results of the $\mathrm{C}$-shape based tag. Time gating parameters are respectively equal to $0.8 \mathrm{~ns}$ and $30 \mathrm{~ns}$ for starting time and ending time. Here again, we can see that the tag response obtained with (11) does not depend on the orientation. Classical reading in copolarization and the proposed method can be used to correctly extract the peak locations (except for the last resonator). Note that for this tag, (11) does not increase the peak magnitude compared to classical reading since for the $\mathrm{C}$-shape resonator, $S_{v v}(0) \gg S_{h h}(0)$ for the first resonators. At higher frequency (after $4 \mathrm{GHz}$ ), as it has been observed for a single element in simulation Fig. 5(a), $S_{v v} \approx S_{h h}$ thus (11) can be higher or lower than the classical method. However, even if (11) is lower than $S_{v v}$, tag ID can sill be decoded. Fig. 11 presents similar results for the loop based tag. Based on the $\mathrm{Q}$ factor of the

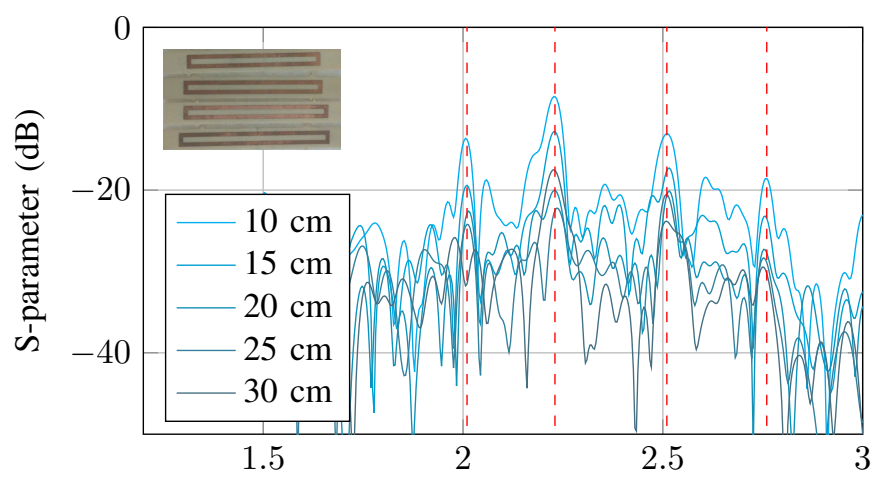

(a) Frequency $(\mathrm{GHz})$

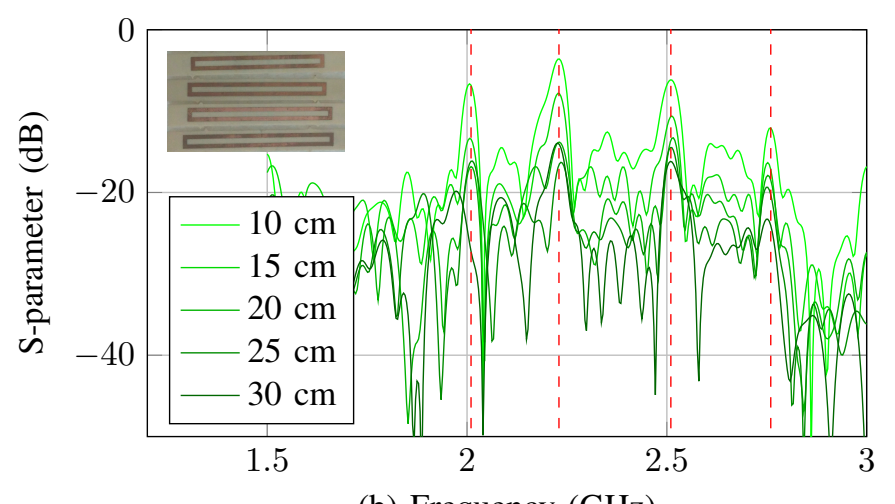

(b) Frequency $(\mathrm{GHz})$

Fig. 12. Measured S-parameters obtained with (a) classical reading with time gating and (b) using (11) with time gating for tag composed of loop resonators at $\theta=45^{\circ}$ as a function of the distance.

scatterers, time gating parameters have been set to $0.2 \mathrm{~ns}$ and $30 \mathrm{~ns}$ for starting time and ending time respectively. Response obtained with (11) is independent from the orientation, and, in that case, increases the peak magnitude since $S_{v v}(0) \approx S_{h h}(0)$ and are added in phase [see Fig. 5(b)] for the loop resonator. Finally, Fig. 9, 10 and 11 show that the proposed method can be used to read most of the scatterers/tags proposed in the literature.

\section{B. Impact of the distance}

This study investigates the performance of the proposed method for real environment measurement with a specific focus on read range. Specifically, the objective is to evaluate the effect of the higher received energy observed in Fig. 9 and 11 over the reading distance of the chipless tag. A loop based tag has been considered here (see Fig. 12) and has been placed in real environment (office) with an orientation of $\theta=45^{\circ}$. Same instruments have been used for the reading (except the semi-anechoic environment) and same time gating parameters (starting time: $2.6 \mathrm{~ns}$, ending time: $30 \mathrm{~ns}$ ) has been used for both readings i.e., $S_{v v}$ and (11). Fig. 12 presents the results for different distances $d$ between the antenna and the tag from $10 \mathrm{~cm}$ to $30 \mathrm{~cm}$ by steps of $5 \mathrm{~cm}$. Received power decreases as the distance increases due to the higher free space loss. Results also show that reading distance remains constant by using (11) since, even if the peak magnitude is higher compared to the classical reading, the (residual) structural 
mode associated with the proposed method after the time gating operation is also higher.

\section{CONCLUSION}

The paper presents a new method allowing to read any chipless tag without any assumption on its orientation. An analytical model is used to justify the approach and is also confirmed in simulations and measurements. Measurements show that magnitude variations of the proposed method is less than $1.74 \mathrm{~dB}$ for all the orientations. Finally, received power at the reader side is increased by the approach since the total energy of the antenna mode can be exploited, maintaining the reading distance compared to classical readings. Last but not least, it has been validated that the proposed approach can be used in real environment.

\section{ACKNOWLEDGMENTS}

This research was funded by the European Research Council (ERC) under the European Union's Horizon 2020 research and innovation program (grant $\mathrm{N}^{\circ} 772539$, ScattererID).

\section{REFERENCES}

[1] M. M. Khan, F. A. Tahir, M. F. Farooqui, A. Shamim, and H. M Cheema, "3.56-bits $/ \mathrm{cm}^{2}$ compact inkjet printed and application specific chipless RFID tag," IEEE Antennas Wireless Propag. Lett., vol. 15, pp. 1109-1112, 2016.

[2] S. Preradovic, I. Balbin, N. C. Karmakar, and G. F. Swiegers, "Multiresonator-based chipless RFID system for low-cost item tracking," IEEE Trans. Microw. Theory Techn., vol. 57, no. 5, pp. 1411-1419, May 2009.

[3] M. Garbati, R. Siragusa, E. Perret, and C. Halopé, "Impact of an IRUWB reading approach on chipless RFID tag," IEEE Microw. Wireless Compon. Lett., vol. 27, no. 7, pp. 678-680, July 2017.

[4] M. A. Islam, Y. Yap, and N. Karmakar, "“ $\Delta$ ' slotted compact printable orientation insensitive chipless RFID tag for long range applications," in 2016 9th International Conference on Electrical and Computer Engineering (ICECE), Dec 2016, pp. 283-286.

[5] A. Vena, E. Perret, and S. Tedjini, "High-capacity chipless RFID tag insensitive to the polarization," IEEE Trans. Antennas Propag., vol. 60, no. 10, pp. 4509-4515, Oct 2012.

[6] M. A. Islam, Y. Yap, N. Karmakar, and A. K. M. Azad, "Orientation independent compact chipless RFID tag," in 2012 IEEE International Conference on RFID-Technologies and Applications (RFID-TA), Nov 2012, pp. 137-141.

[7] M. Martinez and D. van der Weide, "Compact slot-based chipless RFID tag," in 2014 IEEE RFID Technology and Applications Conference (RFID-TA), Sep. 2014, pp. 233-236.

[8] N. Tariq, M. A. Riaz, H. Shahid, M. J. Khan, M. S. Khan, Y. Amin, J. Loo, and H. Tenhunen, "Orientation independent chipless RFID tag using novel trefoil resonators," IEEE Access, vol. 7, pp. 122398 $122407,2019$.

[9] F. Babaeian and N. Karmakar, "Development of cross-polar orientationinsensitive chipless RFID tags," IEEE Trans. Antennas Propag., pp. 1-1, 2020.

[10] O. Rance, N. Barbot, and E. Perret, "Design of planar resonant scatterer with roll invariant cross polarization," 2020, in press.

[11] M. Garbati, A. Ramos, R. Siragusa, E. Perret, and C. Halopé, "Chipless RFID reading system independent of polarization," in 2016 IEEE MTT-S International Microwave Symposium (IMS), May 2016, pp. 1-3.

[12] S. Genovesi, F. Costa, F. A. Dicandia, M. Borgese, and G. Manara, "Orientation-insensitive and normalization-free reading chipless RFID system based on circular polarization interrogation," IEEE Trans. Antennas Propag., vol. 68, no. 3, pp. 2370-2378, March 2020.

[13] A. Vena, E. Perret, and S. Tedjni, "A depolarizing chipless RFID tag for robust detection and its FCC compliant UWB reading system," IEEE Trans. Microw. Theory Techn., vol. 61, no. 8, pp. 2982-2994, Aug 2013.

[14] E. F. Knott, J. F. Shaeffer, and M. T. Tuley, Radar cross section. SciTech Publishing, Inc., 2004.
[15] A. Mackay, "Proof of polarisation independence and nonexistence of crosspolar terms for targets presenting $n$-fold $(n>2)$ rotational symmetry with special reference to frequency-selective surfaces," Electronics Letters, vol. 25, no. 24, pp. 1624-1625, Nov 1989.

[16] S. H. Bickel, "Some invariant properties of the polarization scattering matrix," Proceedings of the IEEE, vol. 53, no. 8, pp. 1070-1072, 1965.

[17] F. Costa, S. Genovesi, and A. Monorchio, "Chipless RFIDs for metallic objects by using cross polarization encoding," IEEE Trans. Antennas Propag., vol. 62, no. 8, pp. 4402-4407, Aug 2014.

[18] A. Vena, E. Perret, and S. Tedjini, "A compact chipless RFID tag using polarization diversity for encoding and sensing," in 2012 IEEE International Conference on RFID (RFID), April 2012, pp. 191-197.

[19] M. A. Islam and N. C. Karmakar, "A novel compact printable dualpolarized chipless RFID system," IEEE Trans. Microw. Theory Techn., vol. 60, no. 7, pp. 2142-2151, July 2012.

[20] A. Vena, E. Perret, and S. Tedjini, "Chipless RFID tag using hybrid coding technique," IEEE Trans. Microw. Theory Techn., vol. 59, no. 12, pp. 3356-3364, Dec 2011.

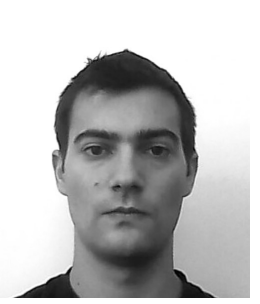

Nicolas Barbot (Member, IEEE) received the M.Sc and $\mathrm{Ph} . \mathrm{D}$. degrees in 2010 and 2013 from the Université de Limoges, Limoges, France.

His $\mathrm{Ph} . \mathrm{D}$. work was focused on error-correcting codes for the optical wireless channel at the Xlim Laboratory, Limoges, France. He also realized a post-doctoral work in joint source-channel decoding at the LSS Laboratory, Gif-sur-Yvette, France. Since September 2014, he has been an Assistant Professor with the Grenoble Institute of Technology, Université Grenoble Alpes, Valence, France. His research interests at the LCIS, Université Grenoble Alpes, include backscattering communications, RFID, and chipless RFID. More recently, he has been investigating the use of chipless tag as low-cost, batteryless, and robust sensors.

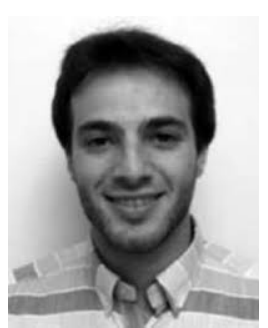

Olivier Rance (Member, IEEE) received the M.Sc. and $\mathrm{Ph} . \mathrm{D}$. degrees in electrical engineering from the Institut National Polytechnique de Grenoble, Grenoble, France, in 2012 and 2017, respectively. He is a Post-Doctoral Fellow with the Institut National Polytechnique de Grenoble. His current scientific interests include leaky-wave antennas, chipless RFID, and RF absorbers. 


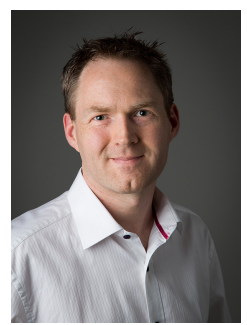

Etienne Perret (Senior Member, IEEE) received the Eng. Dipl. degree in electrical engineering from the École nationale supérieure d'électrotechnique, d'électronique, d'informatique, d'hydraulique et des télécommunications, Toulouse, France, in 2002, and the M.Sc. and Ph.D. degrees in electrical engineering from the Toulouse Institute of Technology, Toulouse, in 2002 and 2005, respectively.

From 2005 to 2006, he held a post-doctoral position with the Institute of Fundamental Electronics, Orsay, France. In 2006, he was appointed as an Associate Professor of electrical engineering with Grenoble INP Institute of Engineering, Université Grenoble Alpes, Valence, France. From 2014 to 2019, he was a Junior Member with the Institut Universitaire de France, Paris, France, an institution that distinguishes professors for their research excellence, as evidenced by their international recognition. From 2015 to 2020, he was an Appointed Member of the French National Council of Universities. He has authored or coauthored over 200 technical conferences, letters and journal articles, and books and book chapters. He holds several patents. His works have generated about 2800 citations. His current research interests include electromagnetic modeling of passive devices for millimeter and submillimeter-wave applications and wireless communications, especially RFID and chipless RFID, and also include advanced computer-aided design techniques based on the development of an automated codesign synthesis computational approach.

Dr. Perret is a Technical Program Committee Member of the IEEE International Conference on RFID. He was a recipient of the French Innovative Techniques for the Environment Award in 2013, the SEE/IEEE Leon Brillouin Award for his outstanding achievement in the identification of an object in an unknown environment using a chipless label or tag in 2016, and the IEEE MTT-S 2019 Outstanding Young Engineer Award. He was awarded an ERC Consolidator Grant in 2017 for his project ScattererID. He was named one of the MIT Technology Review's French Innovators Under 35 for his work on chipless RFID in 2013. He was a keynote speaker and the chairman of several international symposiums. 